\title{
Molecular dynamics simulation of water and ion permeation across silica nanopores in the context of silicate weathering
}

\author{
BASTIEN WILD, CLAIRE WHITE AND IAN BOURG
}

Princeton University

Presenting Author: bwild@princeton.edu

Water-filled silica nanopores play a number of significant roles in fundamental and applied geochemistry, notably regarding mineral weathering or geological nuclear waste disposal, as alteration layers forming at the solid-fluid interface can strongly modulate apparent dissolution rates in a wide variety of natural environments. These systems also have been extensively examined in fundamental studies of the distinct chemical properties of water under nano-confinement. However, relatively few studies have attempted to directly observe the distinct transport properties of water and solutes in such nanopores. Here, we present a series of non-equilibrium molecular dynamics (NEMD) simulations designed to examine the transport of water and ions through uncharged and charged cylindrical silica nanopores as a function of the applied pressure gradient. We determined "bulk" semi-permeable membranes properties of the system, such as permeability and salt rejection capabilities, while examining the details of water structure and solute coordination and their impacts on transport properties. We interpret our results in the context of theoretical models of the anomalous transport properties of nanoporous media and thermodynamic representations of the coupled fluxes of solvent, solute, and electric current. We discuss how these results can support the development of emerging frameworks to incorporate molecular-scale phenomena into continuum-scale transport models, thereby contributing to fill the gap between coupled physical and chemical processes at the atomic scale and behaviors observed at the micro- to macro-scales. 